\title{
Personalized Cancer Care: Risk Prediction, Early Diagnosis, Progression, and Therapy
}

\author{
Kurt S. Zänker ${ }^{a} \quad$ Anne-Lise Borresen-Dale ${ }^{b}$ Hans-Peter Huber ${ }^{c}$ \\ anstitute of Immunology and Experimental Oncology, Center for Biomedical Education and \\ Research, University Witten/Herdecke, Witten, Germany; ${ }^{\text {b} I n s t i t u t e ~ f o r ~ C a n c e r ~ R e s e a r c h, ~}$ \\ The Norwegian Radium Hospital, Oslo University Hospital, Oslo, Norway; ${ }^{c}$ Fritz-Bender \\ Foundation, Munich, Germany
}

\section{What Is It about?}

This meeting report is a follow-up progress report from a previous one in 2013. It summarizes at the cutting edge how cancer manifests in the human body. Basic scientists and clinicians shared their views on personal cancer care and precision cancer medicine with special reference to epigenetic, genetic, and novel therapy strategies.

\section{Key Words}

Risk prediction · Early diagnosis · Progression · Therapy

\begin{abstract}
At the annual prestigious International Symposium of the Fritz-Bender Foundation, Munich, 18-20 May, 2016, researchers, clinicians, and students discussed the state of the art and future perspectives of genomic medicine in cancer. Genomic medicine (also known as precision medicine/oncology) should help clinicians to provide a more precise diagnosis and therapy in oncology for individual patients. The meeting focused on next-generation sequencing methods, analytical computational analysis of big data, and data mining on the way to translational and evidence-based medicine. The meeting covered the social and ethical impact of genomic medicine as well as news and views on antibody targeting of intracellular proteins, on the architecture of intracellular proteins and their impact on carcinogenesis, and on the adaptation of tumor therapy in due consideration of tumor evolution. Subheadings like "Genetic Profiling of Patients and Risk Prediction," "Molecular Profiling of Tumors and Metastases," "Tumor-Host Microenvironment Interaction and Metabolism," and "Targeted Therapy" were subsumed under the main heading of "Personalized Cancer Care."
\end{abstract}




\section{Introduction}

The worldwide importance of precision medicine/oncology was stressed by US President Obama when he announced the Precision Medicine Initiative ${ }^{\circledR}$ in his State of the Union address on January 20, 2015.

The Fritz-Bender Foundation International Symposium Series was continued at the Holmenkollen Park Hotel, Oslo, in May 2016. The authors of the present meeting report chaired the symposium. The theme was "Personalized Cancer Care," and it was a follow-up of a previous meeting held in September 2012 in Oslo [1]. Most of the speakers showed up again in 2016 and offered an update on the progress and newly arising views in their field of expertise.

The symposium was made possible owing to the joint sponsorship of the Fritz-Bender Foundation, Oslo University Hospital, Radium Hospital Foundation, Kreftforeningen, European Association for Cancer Research, The Research Council of Norway, and Life Science, University of Oslo.

The ultimate goal of personalized cancer care is to analyze the biological and genetic signature of cancer cells and to target genetic abnormalities with drug combinations at the right time and with the adequate dosages. In addition, there is the vision of being able to track the therapy precisely with clinical and molecular biomarkers. At present, the promising paradigm only applies to certain cancer types and, therefore, to small numbers of patients. However, the enormous progress in the genomic profiling of cancers, the development of even more powerful data mining techniques, and the possibility to create personal data clouds are fundaments for "precision medicine," also known as "genomic medicine," which are newly coined terms to replace "personalized medicine."

More than 250 delegates were present, and 80 posters were presented. Each talk was followed by lively discussions. The conference brought together highly respected basic scientists, clinicians, and practitioners with junior scientists and students to discuss on a multidisciplinary level the impact of precision medicine/oncology. There were too many excellent presentations at this symposium, and not all can be covered in this report. But this summary fairly reflects the present-day knowledge and new visions to further animate "precision medicine" for the wellbeing of healthy individuals and patients.

In his keynote lecture, Leroy Hood (Institute for Systems Biology, Seattle, WA, USA) expanded on the challenges that traditional approaches to biomedical and pharmaceutical innovation face, which have patently failed to make optimal use of the knowledge available in biomedical science. He introduced the term "systems medicine," which analyzes the dynamic data cloud that surrounds each patient and uses an individualized algorithm that can improve wellness and/or avoid disease. Once 30,000 individual data clouds have been analyzed, they can be aggregated into groups that are relevant to drug responsiveness or other features, and this analysis is based on each individual and not on an averaged population [2]. His remarks focused on a diversification of the former "P4 Medicine" towards (1) pro-active versus reactive, (2) individual versus population, and (3) wellness and disease versus only disease; in this context, he introduced the 108 Person Wellness Project, bridging the gap between wellness and disease; increasing wellness indeed means prevention [3].

In essence, integrative analysis, mining, and computer modeling of big data sets facilitated the development of predictive, preventive, and personalized medicine. 


\section{Genetic Profiling of Patients and Risk Prediction}

Bruce Ponder (Cancer Research UK Cambridge Institute, Cambridge, UK) looked for better ways of classifying single-patient samples by regulators of genetic risk of breast cancer identified by integrative network analysis [4]. A breast cancer gene regulatory network was established comprising transcription factors and groups of putative target genes (regulons), which allowed to examine whether specific regulons are enriched for genes associated with risk loci via expression quantitative trait loci. Bruce Ponder and his team identified 36 overlapping regulons that were enriched for risk loci, forming a distinct cluster within the network. The risk transcription factors driving these regulons are frequently mutated in cancer; 5 transcription factors were identified which are in the midst of the regulon network: forkhead box protein A1 (FOXA1), transacting T-cell-specific transcription factor GATA-3, which regulates luminal epithelial cell differentiation in the mammary gland, pituitary tumor-transforming 1 (PTTG1), which increases the expression of integrin $\alpha 4$ and integrin $\beta 1$, SAM pointed domain containing ETS transcription factor, which is a suitable target for developing specific cancer therapies, and estrogen receptor 1, a ligand-activated transcription factor composed of several domains for hormone binding. Such a network approach provides a platform for determining the regulatory circuits governing breast cancer targets for intervention.

In essence, several lines of evidence support the identification of 5 master regulators and regulons linked to breast cancer risk.

Paul Pharoah (University of Cambridge, Cambridge, UK) reported from the Ovarian Cancer Association Consortium on germline polymorphisms within an enhancer of PSIP1 associated with progression-free survival in epithelial ovarian cancer [5]. The identification of germline single-nucleotide polymorphisms (SNPs) that contribute to variations in individual responses to chemotherapy was carried out using a multi-phase genome-wide association study approach in 1,244 women. The consortium identified 2 SNPs (rs7874043 and rs72700653) in TTC39B associated with progression-free survival. Paul Pharoah also said that the lifetime risk for ovarian cancer lies between 15 and $40 \%$, and, of course, the relative percentage of risk depends on the presence of different risk factors: age, inherited gene mutation (BRCA1 and BRCA2), estrogen hormone replacement therapy, age when menstruation started and ended, never being pregnant, fertility treatment, smoking, use of an intrauterine device, and polycystic ovary syndrome. In this context, he discussed the issue of riskreducing surgery for ovarian carcinoma.

In essence, multiple SNPs at chromosomal position 19p13 indicate common mechanisms underlying breast and ovarian cancer risk.

\section{Molecular Profiling of Tumors and Metastases}

Elaine Mardis (McDonnell Genome Institute, Washington University School of Medicine, St. Louis, MO, USA) continued the focus on genetic profiling and put forward the question what is next for genetics and genomics of precision medicine? It is well established that the immune system can recognize cancer cells and that immune-directed interventions can induce tumor regression. The translation of big data obtained from in vitro and in vivo experiments does not clinically lead to the expected results. The newest advances in next-generation sequencing and epitope prediction now permit the rapid identification of mutant tumor neoantigens [6]. These mutant tumor neoantigens and the derived diverse peptide motifs can be used to activate specific CD8-positive T cells, when T or B cells recognize these aberrant protein domains presented by cognate dendritic cells. In the future, this approach is a fast and precise way for a therapeutic cancer vaccine $[7,8]$. Elaine Mardis presented a "window of 
opportunity" for clinical trials in precision medicine starting from an "'N of 1 ' to $\mathrm{N}$ of more" perspective [9]. A comprehensive genomic analysis revealed a successful therapy strategy for a patient with relapsed adult B-lymphoblastic leukemia [10]. The transcriptome analysis identified aberrant overexpression of the protein kinase encoded by FLT3 gene. Sunitinib rapidly induced a near-complete remission, permitting the patient to proceed to a matchedunrelated donor stem cell transplantation, and the patient stayed in complete remission for more than 4 years. Elaine Mardis wrapped up the session by saying: "Precision medicine is going to be brilliant, and it is now."

In essence, genomics contribute to personalized vaccine development efforts by identifying tumor-specific neoantigens which stimulate immunity against cancer cells.

Peter van Loo (Francis Crick Institute, London, UK) studies the subclonal architecture of cancers. He presented data from collaborative work unravelling the life history of cancer genomes based on massively parallel sequencing data. He and his team anticipate that a largesale pan-cancer approach would give profound insights into carcinogenesis and cancer evolution. For this approach, they coined the term "molecular archaeology of cancer." In an ongoing pan-cancer analysis of 2,800 tumors, they are gaining insights into the extent of heterogeneity in different tumors, the magnitude of acquisition of genetic alterations, and the evolution of mutational signatures. In lethal metastatic prostate cancer, his group showed for the first time polyclonal seeding, challenging the concept that each metastasis is formed by 1 disseminating cancer cell [11].

In essence, derived from the context of androgen-deprived metastatic prostate cancer, there is evidence for the existence of polyclonal seeding in human malignancy and a clonal relationship between different metastases.

Charles M. Perou (UNC School of Medicine, Chapel Hill, NC, USA) introduced the genomic analysis of immune cell infiltrates across 11 tumor types [12]. Immune infiltration of the tumor microenvironment has been associated with improved survival for some patients with solid tumors. Quantitative genomic data sets obtained by the analysis of heterogeneous immune infiltrates are prognostic markers because these signatures of $\mathrm{T}$ and $\mathrm{B}$ cells and of macrophages are likely to predict overall survival. Therefore, genomic analysis of immune cell infiltrates might serve to identify potential biomarkers of response to cancer immunotherapy.

In essence, an adaptive immune gene expression profile exists in genomic subtypes of breast and ovarian cancer.

\section{Tumor-Host Microenvironment Interaction and Metabolism}

The classification of tumors based on genetic signatures is one side of the coin, but the time has come to merge tumor cell-centric genomics with the microenvironment of tumor cells, another important hallmark of cancer. Morag Park (McGill University, Montréal, QC, Canada) introduced a framework for building genome-wide epithelial-stromal co-expression networks composed of pairwise co-expression relationships between mRNA levels of genes in the epithelium and stroma across a population of patients [13]. Tumor-associated stroma is different from normal stroma with respect to gene expression and predicts tumor therapy outcome in breast cancer. She introduced the term "immune cold," where immune-competent cells are either (1) tumor margin restricted and/or (2) tumor stroma restricted versus tumor permissive. The tumor cell-microenvironment architecture is a morphological regulator to facilitate tumor-permissive immune cell invasion. She showed that the immune gene expression signature correlates with the outcome in triple-negative breast cancer, and, e.g., a 
stromal signature of fibrosis strongly influences the CD8-positive cell localization within a tumor tissue.

In essence, epithelial-stromal cross-talks linked to immune competence play an essential role in invasive breast cancer.

Mina Bissell (Lawrence Berkeley National Laboratory, Berkeley, CA, USA) gave, as always, a vibrant and fascinating talk, summarizing the last 10 years of her very productive work. Her lab developed 3D culture conditions for human breast epithelial cells [14], and the results suggest that the phenotype is dominant over the genotype not only in normal but also in cancer cells. A key feature of all luminal breast epithelial cells is the basoapical axis. Transmembrane integrins at the basal side of cells serve as anchorage points and receptors for the basal membrane components. They trigger intracellular signaling and participate in the perception of the cell's microenvironment. There is a link between the extracellular matrixcontrolled cell shape and chromatin structure, and this link is mediated by changes in the actin cytoskeleton, depending on at which cell poles actin and laminin are located. Interestingly, inhibitors of Rho kinase (ROCK) signaling revert the malignant phenotype of breast cancer cells in a 3D context. Inhibition of ROCK decreases EGFR and integrin $\beta 1$ levels and, in consequence, suppresses activation of Akt, MAPK, and FAK as well as GLUT3 and LDHA levels. These effects were not seen in 2D cultures [15]. With respect to GLUT3, she showed for the first time that the cellular architecture of the mammary gland determines the glucose utilization. Low glucose level can revert a malignant phenotype. In this context, she promotes centers of excellence for training the next generation of biomedical investigators in the glycosciences [16].

In essence, there is a need for complex 3D culture models to unravel novel pathways in the genomic era and to identify robust biomarkers in breast cancer.

\section{Targeted Therapy}

Joe Gray (Oregon Health \& Science University, Portland, OR, USA) spoke about a major challenge concerning the emerging precision medicine. He stated that it is necessary to make links between functional oncogene signatures of cancer subsets and combinations of drugs that can be used to guide the treatment of individual patients to improved outcomes and decreased toxicity [17]. Joe Gray gave an example of large-scale drug screening by defining a molecular signature, which may guide therapeutic approaches for tumors with high mitotic network activity [18]. This approach is supported by Seashore-Ludlow et al. [19] who developed a novel annotated cluster multidimensional enrichment analysis to explore the association between groups of small molecules and groups of cancer cell lines in a new, quantitative, sensitive data set. Functional oncogene signatures may guide the design of combination therapies and avoid toxicities that have been associated with the combined use of multiple-targeted agents.

In essence, genome co-amplification upregulates a mitotic network activity that predicts outcome and response to small molecular inhibitors of the mitotic machinery in breast cancer.

René Bernards (Center for Biomedical Genetic and Cancer Genomics Center, The Netherlands Cancer Institute, Amsterdam, The Netherlands) delivered the European Association for Cancer Research lecture entitled: "A Vulnerability of BRAF-Resistant Melanoma with Potential Clinical Utility." Treatment of BRAF ( $p . V 600 E$ )-mutant melanoma by small-molecule drugs that target the BRAF or MEK kinases can be effective, but resistance develops invariably [20]. René Bernards' group found that melanoma cells with low SOX10 and high EGFR expression gain a benefit for proliferation from small-molecule drugs directed at BRAF or MEK. But this is reversed when the drug treatment is discontinued. He suggested that with a "drug holiday," 
otherwise BRAF or MEK inhibitor-resistant melanoma patients may regain sensitivity to these drugs.

In essence, signals can emanate from chemically induced tumor regression itself, thereby providing a local microenvironment that is conductive for proliferation and metastasizing of specific subclones within the regressing tumor population.

Alberto Bardelli (Department of Oncology, University of Torino, Italy) began his talk by saying, "today's science is tomorrow's medicine," and that targeted therapies must take into account the continuous evolution of cancer cells, which means that therapeutic strategies have to be adapted to tumor evolution. He introduced the idea of "evolutionary thinking of cancer" within therapy concepts. It quickly became apparent that patients treated with single agents always develop resistance within months after initiating therapy because subclones are selected that are capable of growing under drug pressure. A possible approach to overcome these limitations of targeted agents is to use drug combinations instead of monotherapies. Tumors should be treated with more agents that hit different targets. The success of this concept has been demonstrated in colorectal cancer with a model of Combo blockade of EFGR-MEK, which triggers Bcl-2 and Mcl-1 downregulation and initiates apoptosis [21].

In essence, drug-tolerant cells without bona fide resistance mechanisms may survive initial drug treatment by epigenetic adaptation and acquire genetic resistance mechanisms over time.

According to Soldano Ferrone (Massachusetts General Hospital/Harvard Medical School, Boston, MA, USA), the application of antibody-based immunotherapy to the treatment of malignant diseases is limited by the low number of clinically relevant tumor antigens which can be targeted by specific antibodies. One restriction is the requirement that molecules (neoantigens) have to be expressed on the plasma membrane; this assumption has excluded intracellular molecules as potential targets. Soldano Ferrone and his collaborators have challenged this assumption since intracellular molecules can traffic to the cell membrane and become available for reaction with antibodies. His group has supported the validity of this assumption by describing the characteristics of the antibody W9 which recognizes an extracellular epitope of the intracellular Grp94 protein, a member of the heat shock protein (HSP) 90 family. Grp94 is expressed on malignant cells present in surgically removed tumors. The W9 antitumor activity can be enhanced by combination with inhibitors of signaling pathways associated with the proliferation and survival of tumor cells. If the results with the antibody W9 can be confirmed by additional antibodies, intracellular antibodies may represent a useful source of targets of antibody-based immunotherapy.

In essence, monoclonal antibody $\mathrm{W} 9$ recognizes an extracellular epitope of the intracellular Grp94 protein to counteract BRAF inhibitor resistance in BRAF ( $p . V 600 E$ ) melanoma.

\section{Translation}

Anne-Lise Borresen-Dale (Institute for Cancer Research, Oslo University Hospital, Oslo, Norway) presented data from breast cancers analyzed by high-throughput technologies at several molecular levels and demonstrated that combining the various profiles derived from each level can further subdivide the initially discovered expression subclasses and improve prognostic potential. The combined analyses of gene regulation at the various levels point to specific biological functions and molecular pathways that are deregulated in breast cancer [22]. She also presented data from the large collaborative effort performing whole-genome sequencing of 560 breast cancers [23]. This study identified 93 protein-coding cancer genes carrying driver mutations. Mutational signature analysis revealed 12 base substitution and 6 rearrangement signatures. These analyses highlight the large repertoire of cancer genes and 
mutational processes operative in breast cancer. Specific alterations identified by such studies can further be analyzed using a combination of immunofluorescence and fluorescence in situ hybridization (FISH) techniques ("double immunoFISH") to identify intratumor heterogeneity in tumors from neoadjuvant-treated patients prior to and after therapy [24]. Results show that the genomic variability prior to therapy was more diverse in the partial responders than in the responders, and the remaining tumor was even more heterogeneous after treatment than prior to treatment.

In essence, a genome-based stratification of breast cancer is important and has important implications for designing therapeutic strategies.

\section{The Impact of Social and Ethic Issues}

The meeting also raised critical issues and questions that address the future of precision medicine. The use of genomics to decipher the molecular signature of the mutational landscapes of cancer is now transitioning from the discovery phase into clinics to navigate patient care. Eva Winkler (National Center of Tumor Diseases, Heidelberg, Germany) discussed the different "-omics" with respect to their application within and outside a clinical context with special reference to the impact of the genetics of an individual on her/his psychological condition as well as the social consequences [25]. Every human genome statistically contains a number of potentially disease-related motifs that may be detected during, e.g., genomewide disease-associated sequencing. She provided evidence that there are fewer incidental findings in clinical research than expected. She argued that filtering techniques and methods of analysis are systemic filters and, therefore, the question arises whether the ethical debate is focused on the right issue [26]. At the moment, big genomic data sets from individuals are subjected to filter techniques that avoid the occurrence of incidental findings.

In essence, formal governance structures are necessary requirements to achieve a balance between the potential of precision medicine and protecting the privacy of the individual.

\section{Summary and Conclusion}

Kurt S. Zänker (University Witten/Herdecke, Witten, Germany) summarized the meeting and provided future perspectives derived from the speakers' conclusions. The enormous progress in biomedical science technologies, which simultaneously measure thousands of data points, as well as sophisticated system biology software, which subjects the plethora of heterogeneous data to data mining, make next-generation diagnosis, biomarker identification, and individual drug delivery in the era of precision medicine possible. Genome medicine, which is part of precision medicine, relies on 2 principles: (1) data generation from integrated biobanks (e.g., evaluation of genetic tumor signatures linked to robust and relevant clinical disease outcome) and (2) prospective individual-level tumor data sets (obtained, e.g., by multiple biopsies, tumor tissue imaging, or single-cell sequencing) that reveal the clonal history and, subsequently, the cellular and (epi-)genetic heterogeneity of a tumor in a singletumor patient. The knowledge from a framework of biobanks combined with timely omicsbased forward strategies are key drivers to focusing therapeutics on individuals rather than on a population. Precision medicine uses (epi-)genetic information from the individual and the tumor to match the right patient, at the right time, with the right treatment. The scientific community comprehends the potential of an individual care; however, the clinical transition process is slow. Moreover, various ethical, legal, and social issues linked to privacy will 
increasingly arise in the postgenomic era. Nevertheless, precision medicine/oncology receives increasing attention due to its realistic promise of improving clinical outcome for individual patients or of defining subsets of patients who will more likely respond to biomarker-driven therapies.

\section{Disclosure Statement}

The authors declare no competing interest.

\section{References}

1 Zänker KS, Mihich E, Huber HP, Borresen-Dale AL: Personalized Cancer Care Conference. J Pers Med 2013;3: 70-81.

-2 Toga AW, Foster I, Kesselman C, Madduri R, Chard K, Deutsch EW, Price ND, Glusman G, Heavner BD, Dinov ID, Ames J, Van Horn J, Kramer R, Hood L: Big medical data as the key resource for discovery science. J Am Med Inform Assoc 2015;22:1126-1131.

-3 Hood L, Lovejoy JC, Price ND: Integrating big data and actionable health coaching to optimize wellness. BMC Med 2015;13:4.

-4 Castro MA, de Santiago I, Campbell TM, Vaughn C, Hickey TE, Ross E, Tilley WD, Markowetz F, Ponder BA, Meyer KB: Regulators of genetic risk of breast cancer identified by integrative network analysis. Nat Genet 2016;48:12-21.

-5 French JD, Johnatty SE, Lu Y, et al; Australian Ovarian Cancer Study Group; Australian Ovarian Cancer Study, Lambrechts D, Despierre E, Van Nieuwenhuysen E, et al; Ovarian Cancer Association Consortium: Germline polymorphisms in an enhancer of PSIP1 are associated with progress-free survival in epithelial ovarian cancer. Oncotarget 2016;7:6353-6368.

6 Johanns TM, Ward J, Wilson C, Kobayashi DK, Bender D, Fu Y, Alexandrov A, Artyomov MN, Miller CA, Mardis ER, Dunn GP: 143 Identification of neoantigen-specific CD8+ T cells in two murine orthotopic glioblastoma models using cancer immunogenomics. Neurosurgery 2016;63(suppl 1):158.

7 Gubin MM, Artyomov MN, Mardis ER, Schreiber RD: Tumor neoantigens: building a framework for personalized cancer immunotherapy. J Clin Invest 2015;125:3413-3421.

-8 Hundal J, Carreno BM, Petti AA, Linette GP, Griffith OL, Mardis ER, Griffith M: pVAC-Seq: a genome-guided in silico approach to identifying tumor neoantigens. Genome Med 2016;8:11.

-9 DeBerardinis RJ, Mardis ER: From "N of 1" to N of more. Cold Spring Harb Mol Case Stud 2015;1:a000521.

10 Griffith M, Griffith OL, Krysiak K, Skidmore ZL, Christopher MJ, Klco JM, Ramu A, Lamprecht TL, Wagner AH, Campbell KM, Lesurf R, Hundal J, Zhang J, Spies NC, Ainscough BJ, Larson DE, Heath SE, Fronick C, O'Laughlin S, Fulton RS, Magrini V, McGrath S, Smith SM, Miller CA, Maher CA, Payton JE, Walker JR, Eldred JM, Walter MJ, Link DC, Graubert TA, Westervelt P, Kulkarni S, DiPersio JF, Mardis ER, Wilson RK, Ley TJ: Comprehensive genomic analysis reveals FLT3 activation and a therapeutic strategy for a patient with relapsed adult B-lymphoblastic leukemia. Exp Hematol 2016;44:603-613.

11 Gundem G, Van Loo P, Kremeyer B, Alexandrov LB, Tubio JMC, Papaemmanuil E, Brewer DS, Kallio HML, Högnäs G, Annala M, Kivinummi K, Goody V, Latimer C, O’Meara S, Dawson KJ, Isaacs W, Emmert-Buck MR, Nykter M, Foster C, Kote-Jarai Z, Easton D, Whitaker HC; ICGC Prostate UK Group, Neal DE, Cooper CS, Eeles RA, Visakorpi T, Campbell PJ, McDermott U, Wedge DC, Bova SG: The evolutionary history of lethal metastatic prostate cancer. Nature 2015;520:353-357.

12 Iglesia MD, Parker JS, Hoadley KA, Serody JS, Perou CM, Vincent BG: Genomic analysis of immune cell infiltrates across 11 tumor types. J Natl Cancer Inst 2016;108:djw144.

13 Oh EY, Christensen SM, Ghanta S, Jeong JC, Bucur O, Glass B, Montaser-Kouhsari L, Knoblauch NW, Bertos N, Saleh SM, Haibe-Kains B, Park M, Beck AH: Extensive rewiring of epithelial-stromal co-expression networks in breast cancer. Genome Biol 2015;16:128.

14 Vidi PA, Bissell MJ, Lelièvre SA: Three-dimensional culture of human breast epithelial cells: the how and the why. Methods Mol Biol 2013;945:193-219.

15 Matsubara M, Bissell MJ: Inhibitors of Rho kinase (ROCK) signaling revert the malignant phenotype of breast cancer cells in 3D context. Oncotarget 2016;7:31602-31622.

16 Agre P, Bertozzi C, Bissell M, Campbell KP, Cummings RD, Desai UR, Estes M, Flotte T, Fogleman G, Gage F, Ginsburg D, Gordon JI, Hart G, Hascall V, Kiessling L, Kornfeld S, Lowe J, Magnani J, Mahal LK, Medzhitov R, Roberts RJ, Sackstein R, Sarkar R, Schnaar R, Schwartz N, Varki A, Walt D, Weissman I: Training the next generation of biomedical investigators in glycosciences. J Clin Invest 2016;126:405-408. 
17 Guest ST, Kratche ZR, Irish JC, Wilson RC, Haddad R, Gray JW, Garrett-Mayer E, Ethier SP: Functional oncogene signatures guide rationally designed combination therapies to synergistically induce breast cancer cell death. Oncotarget 2016;7:36138-36153.

18 Hu Z, Mao JH, Curtis C, Huang G, Gu S, Heiser L, Lenburg ME, Korkola JE, Bayani N, Samarajiwa S, Seoane JA, Dane MA, Esch A, Feiler HS, Wang NJ, Hardwicke MA, Laquerre S, Jackson J, Wood KW, Weber B, Spellman PT, Aparicio S, Wooster R, Caldas C, Gray JW: Genome co-amplification upregulates a mitotic gene network activity that predicts outcome and response to mitotic protein inhibitors in breast cancer. Breast Cancer Res 2016;18: 70 .

19 Seashore-Ludlow B, Rees MG, Cheah JH, Cokol M, Price EV, Coletti ME, Jones V, Bodycombe NE, Soule CK, Gould J, Alexander B, Li A, Montgomery P, Wawer MJ, Kuru N, Kotz JD, Hon CS, Munoz B, Liefeld T, Dančík V, Bittker JA, Palmer M, Bradner JE, Shamji AF, Clemons PA, Schreiber SL: Harnessing connectivity in a large-scale smallmolecules sensitivity dataset. Cancer Discov 2015;5:1210-1223.

20 Sun C, Wang L, Huang S, Heynen GJ, Prahallad A, Robert C, Haanen J, Blank C, Wesseling J, Willems SM, Zecchin D, Hobor S, Bajpe PK, Lieftink C, Mateus C, Vagner S, Grernrum W, Hofland I, Schlicker A, Wessels LF, Beijersbergen RL, Bardelli A, Di Nicolantonio F, Eggermont AM, Bernards R: Reversible and adaptive resistance to BRAF (V600E) inhibition in melanoma. Nature 2014;508:118-122.

-21 Misale S, Bozic I, Tong J, Peraza-Penton A, Lallo A, Baldi F, Lin KH, Truini M, Trusolino L, Bertotti A, Di Nicolantonio F, Nowak MA, Zhang L, Wood KC, Bardelli A: Vertical suppression of the EGFR pathway prevents onset of resistance in colorectal cancers. Nat Commun 2015;6:8305.

-22 Kristensen VN, Lingjaerde OC, Russnes HG, Vollan HK, Frigessi A, et al: Principles and methods of integrative genomic analyses in cancer. Nat Rev Cancer 2014;14:299-313.

23 Nik-Zainal S, Davies J, Staaf J, Ramakrishna M, Glodzik D, Zou X, Martincorena I, Alexandrov LB, Martin M, Wedge D, Van Loo P, et al: Landscape of somatic mutations in 560 breast cancer whole-genome sequences. Nature 2016;534:47-54.

24 Trinh A, Rye IH, Almedro V, Helland Å, Russnes HG, Markowetz F: GoIFISH: a system for the quantification of single cell heterogeneity from FISH images. Genome Biol 2014;15:442.

-25 Winkler EC, Wiemann S: Findings made in gene panel to whole genome sequencing: data, knowledge, ethics - and consequences? Expert Rev Mol Diagn 2016;16:1259-1270.

26 Schuol S, Schickhardt C, Wiemann S, Bartram CR, Tanner K, Eils R, Meder B, Richter D, Glimm H, von Kalle C, Winkler EC: So rare we need to hunt for them: reframing the ethical debate in incidental findings. Genome Med 2015;7:83. 